\title{
20 años de entrenamiento cognitivo: una perspectiva amplia
}

\author{
Melina Vladisauskas ${ }^{1 *}$, Andrea P. Goldin ${ }^{1 \star}$ \\ 'Universidad Torcuato Di Tella, CONICET, Laboratorio de Neurociencia, Buenos Aires, Argentina.
}

\begin{abstract}
Resumen
La posibilidad de entrenar ciertas capacidades de nuestra mente para mejorarlas ha sido motivo de un sinfín de discusiones en el área de las neurociencias cognitivas en los últimos 20 años. Este ferviente debate continúa acumulando publicaciones académicas con mensajes radicalmente opuestos, y alcanzar un consenso no parece cercano. ¿Es el entrenamiento cognitivo una poderosa herramienta igualadora de oportunidades? ¿O ya ha llegado la hora de dejar de invertir en las seductoras, pero falsas, promesas de herramientas para "mejorar nuestro cerebro"?

En este trabajo se fundamentan ambas partes de la discusión. Se concluye con una perspectiva propia que permite explicarlas como producto de nuestra experiencia, que desarrolla e implementa un software de libre acceso para entrenar la cognición infantil a través del juego.
\end{abstract}

apgoldin@gmail.com

Citación:

Vladisauskas M, Goldin AP. 20 años de entrenamiento cognitivo: una perspectiva amplia JONED. Journal of Neuroeducation. 2020; 1(1); $130-135$

doi: $10.1344 /$ joned.v1i1.31628

Editora:

Laia Albó Pérez (Universitat Pompeu Fabra, España)

Revisores:

Gérardo Restrepo (Université

Sherbrooke, Canadá) y Enrique

Casillas (Universidad Marista de

Guadalajara)

El manuscrito ha sido aceptado por todos los autores, en el caso de haber más de uno, y las figuras, tablas e imágenes no están sujetos a ningún tipo de Copyright.

\begin{abstract}
Over the last 20 years, the possibility of improving cognitive skills by training has been the subject of endless discussions in the Cognitive Neurosciences community. Publications with opposed messages accumulate and, despite the huge amount of data collected to date, there is no consensus around this subject. Is Cognitive training a powerful equalizer for society? Or is it time to stop investing resources in the seductive, but false, promises of tools to "improve the brain"?

In this Perspective, both sides of the debate are described and analysed. We explain why we have not reach consensus yet after our experience developing and implementing a free software aimed to train cognitive processes in children through play.
\end{abstract}

Palabras clave: estimulación cognitiva, videojuegos, funciones ejecutivas, niños, transferencia. 


\section{Introducción}

El control consciente de nuestro comportamiento y nuestras acciones se logra gracias a un conjunto de herramientas cognitivas conocidas en la literatura como funciones ejecutivas (FE). Son esenciales para desenvolvernos de forma exitosa en todo tipo de actividades voluntarias y en situaciones que presentan nuevos desafíos a los cuales debemos adaptarnos, desde tomar notas en una clase hasta planear los destinos en unas vacaciones o simplemente una breve compra en el almacén'.

Numerosos estudios han mostrado que las FE poseen un elevado carácter predictor de la preparación escolar, del comportamiento en el aula, del logro académico, del desarrollo de la lectoescritura y el pensamiento matemático en los primeros grados y de aspectos más amplios, como la calidad de vida y la salud física ${ }^{2-9}$. Su desarrollo comienza a edades muy tempranas y está determinado por dos factores que dan origen a las características de toda la diversidad de los organismos vivos: los genes y el ambiente. No es objeto de este trabajo analizar los factores genéticos ni su interacción con el desarrollo de las FE (tópico abordado en, por ejemplo, ${ }^{10}$ $\left.y{ }^{11}\right)$. En este artículo partimos de la base de que el desarrollo individual de las FE integra factores sociales y que la existencia de riesgo de vulnerabilidad social ${ }^{12}$, de adversidad temprana ${ }^{13} \mathrm{O}$ de estrés ${ }^{14,15}$ pueden tener efectos negativos sobre su desarrollo.

Así como las condiciones ambientales desfavorables tienen un impacto negativo en las $\mathrm{FE}$, ¿existirán condiciones favorables que ayuden a mejorarlas? ${ }^{16-18}$ Una posible aproximación a la solución de este problema surgió en el campo de las neurociencias. La estimulación cognitiva se beneficia de la plasticidad neural para mejorar o mantener distintas capacidades mentales ${ }^{19,20}$. Así como una deportista puede correr en la cinta para mejorar su capacidad pulmonar $y$, consecuentemente, su rendimiento, en el fútbol, por ejemplo, entrenar las FE podría ayudar a mejorarlas y que, a la vez, esas mejoras impacten positivamente en otros aspectos no entrenados de forma directa (fenómeno conocido como transferencia, que se clasifica como cercana o lejana según la similitud que exista entre la habilidad entrenada y las no entrenadas).

En los últimos 20 años, numerosos grupos de investigación comenzaron a diseñar e implemen- tar propuestas de intervención basadas en la comprensión del funcionamiento de la mente buscando lograr transferencia. Estos programas de investigación confirmaron que se logró un impacto positivo en el desarrollo cognitivo en diferentes contextos de aprendizaje y en distintas poblaciones (incluyendo también a niños con Trastorno de deficiencia de atención e hiperactividad -TDAH- o con dislexia, adultos mayores y pacientes con Alzheimer, entre otros) ${ }^{20-25}$.

Una gran parte de estos estudios incluye evaluaciones del impacto de los entrenamientos en medidas de transferencia tomadas en el laboratorio. Sin embargo, el objetivo final deseable es mejorar las FE y que esas mejoras puedan redundar en actividades cotidianas, como el aprendizaje y el rendimiento académico. En otras palabras, la intención del entrenamiento cognitivo no es solo mejorar el rendimiento en la tarea en la que se entrenaron o en otras muy similares (transferencia cercana), sino que esas mejoras se puedan generalizar en tareas con exigencias distintas (transferencia lejana).

A pesar de los numerosos esfuerzos por comprender el funcionamiento y el alcance de los protocolos de entrenamiento de FE, la comunidad científica aún no ha llegado a un consenso. La principal duda está puesta en torno a si ciertas mejoras, producto del entrenamiento, pueden impactar en otros aspectos no entrenados. En otras palabras, ¿son las FE tan maleables como para producir transferencia lejana a través de su entrenamiento explícito? En este trabajo presentamos un estado actualizado en torno a este debate dentro de la comunidad científica y planteamos nuestro punto de vista sobre por qué no se ha alcanzado aún un consenso.

\section{Evidencias a favor del entrenamiento}

Desde el surgimiento de la idea de que las FE podrían ser entrenadas, muchos protocolos de estimulación comenzaron a ponerse a prueba en intervenciones diversas. Dado que estos protocolos no se caracterizan por ser particularmente homogéneos entre $s^{26}$, la diversidad de resultados reportados es bastante grande; por eso, su eficiencia continúa siendo puesta en duda en la comunidad científica (en la sección siguiente analizamos esto en profundidad).

Muchos grupos de investigación se han centrado en estos años en desarrollar y llevar a cabo intervenciones enfocadas en el entrenamiento de una FE 
específica en particular: la memoria de trabajo (MT, la capacidad de retener en mente y manipular información durante un período de tiempo muy corto a fin de completar una tarea específica ${ }^{27}$ ). Aunque no es la única FE fundamental para el éxito en tareas académicas muy frecuentes, como tomar notas en una clase o resolver cálculos aritméticos, la estimulación de esta FE ha recibido particular atención en el diseño de distintos entrenamientos. Producto de diversos protocolos de estimulación enfocados, en la MT se ha observado transferencia lejana a otras habilidades, como las capacidades de razonar lógicamente y de resolver problemas novedosos ${ }^{28,29}$. Específicamente en el ámbito escolar, mejoraron la adquisición del lenguaje y el razonamiento matemático $0^{8,18,30,31}$. Incluso se evidenciaron beneficios durante ocho meses en el funcionamiento cotidiano en niños y niñas con TDAH después de un entrenamiento de cinco semanas ${ }^{32}$.

Todo ello sugiere que el entrenamiento enfocado en un único aspecto de la cognición produce mejoras que solo se transfieren a otras tareas muy similares. Sin embargo, la implementación de protocolos que ejercitan más de una FE a la vez muestra resultados prometedores hacia la transferencia lejana ${ }^{33}$. Nuestro grupo de investigación, por ejemplo, no solo encontró mejoras en tareas no entrenadas ${ }^{34,35}$, sino en el rendimiento académico de niños en edad escolar y en situación de vulnerabilidad social después del entrenamiento, lúdico y en sus propias escuelas, y de varias $\mathrm{FE}$ en simultáne ${ }^{36}$. Más aún, en un metanálisis de 2016 proponen que aquellos programas de entrenamiento que mayor impacto y transferencia lejana muestran son los que intentan, además, reducir el peso de los factores que impactan negativamente en las FE (como el estrés, la exclusión social o la mala salud física) ${ }^{37}$. La gran crítica con respecto a estos estudios es que, al estimular simultáneamente muchos aspectos, el hecho de encontrar mejoras posteriores no permite dilucidar cuál o cuáles fueron las causas de esta mejora.

\section{Evidencias en contra del entrenamiento}

Un estudio que comenzó a poner en duda el funcionamiento de los protocolos de entrenamiento cognitivo y, por ende, la existencia de la transferencia lejana fue un entrenamiento online realizado en $2010^{38}$, en el que participaron más de 10000 adultos. Los resultados mostraron mejoras inmediatas de los sujetos en todas las tareas entrenadas, pero no encontraron impacto en habilidades no entrenadas. Este estudio fue publicado en una prestigiosa revista y recibió numerosas críticas metodológicas ${ }^{21,39,40}$, por ejemplo, que cada participante decidía cuántas sesiones jugar y qué capacidades entrenar $y$, justamente, esa poca sistematicidad puede explicar la escasa transferencia.

Carece de sentido probar la inexistencia de la transferencia lejana implementando un protocolo específico cuando aún no sabemos cuáles son las condiciones para que exista transferencia. Por eso, las investigaciones posteriores tomaron otro rumbo. En los siguientes años se realizaron numerosos metanálisis estudiando los resultados de la literatura. Con relación al entrenamiento de MT ya mencionado, por ejemplo, diversos metanálisis critican seriamente la existencia de una transferencia lejana a otros dominios cognitivos ${ }^{41,42}$, y alegan que los estudios que la apoyan presentan sesgos de inclusión o no diferencian diseños experimentales que incluyeron grupos de control activos de los que incluyeron controles pasivos.

Otro resultado reiterado de los trabajos metanalíticos apunta a las diferencias entre los diseños experimentales. En un estudio de 2015 se evalúa la existencia de transferencia lejana utilizando algunas características del entrenamiento como moduladores del análisis, y encuentran que la duración de cada sesión y el largo de la intervención explican las diferencias observadas en el impacto de los entrenamientos ${ }^{43}$. En otro estudio experimental reciente ${ }^{44}$ se encuentra un efecto placebo relacionado con la expectativa de mejorar la cognición: se observaron mejoras en aquellos individuos que esperaban que un falso entrenamiento les resultara beneficioso. Es importante resaltar que en ninguno de los estudios anteriores se evidenció transferencia lejana: los efectos observados y comparados con respecto a los factores analizados se produjeron en tareas similares a las entrenadas.

Si bien muchos de los trabajos mencionados recomiendan dejar de invertir recursos en desarrollar protocolos de entrenamiento dada la falta de evidencia de transferencia lejana ${ }^{45,46}$, muchos otros de los citados proponen interesantes mejoras metodológicas para poder terminar de resolver la controver$\mathrm{sia}^{37,43,44,47,48}$. 


\section{Acercando posiciones}

Una buena representación del debate acerca de la existencia de la transferencia producto del entrenamiento cognitivo ocurrió en 2014, cuando un grupo internacional de más de 70 investigadores publicó una carta abierta argumentando que no existe evidencia científica de que los protocolos de entrenamiento cognitivo funcionen ni que prevengan el deterioro de las FE que se produce por la edad ${ }^{49}$. Meses después, un grupo formado por casi el doble de investigadores publicó otra carta abierta en respuesta a ello planteando que la literatura científica contiene numerosas evidencias de que el entrenamiento cognitivo tiene una gran variedad de efectos positivos tanto en tareas de laboratorio como cotidianas ${ }^{50}$.

En un esfuerzo por reunir toda la evidencia disponible proveniente de los distintos protocolos existentes de entrenamiento cognitivo, se organizó una gran base de datos online que contenía estudios que muestran que el entrenamiento cognitivo computarizado tiene efectos positivos sobre la cognición ${ }^{51}$. A esto se le suma un listado de criterios que cabe tener en cuenta para la implementación individual, o en instituciones como escuelas o geriátricos, de un protocolo de entrenamiento. Esos criterios surgen a partir de las fallas metodológicas que fueron demostradas anteriormente, como la falta de un grupo control activo o la existencia de un efecto placebo. Los esfuerzos por mejorar los protocolos de entrenamiento y mediciones comportamentales continúan; recientemente un grupo de 50 científicos publicó un trabajo que incluye recomendaciones en distintos tópicos, como la implementación de grupos control activos o el reporte de los resultados y su posterior comunicación ${ }^{52}$.

La vigencia de este debate muestra que, contrariamente a lo esperado y dada la cantidad de estudios de entrenamiento cognitivo realizados, esta área de investigación se encuentra aún en una fase exploratoria. Se desconocen cuestiones fundamentales necesarias para que un aspecto cognitivo se bene- ficie de un entrenamiento puntual, como la duración ideal, o incluso mínima y máxima, de las sesiones de entrenamiento y su prolongación en el tiempo, la frecuencia de estimulación, el peso de la motivación para jugar y el límite en las definiciones de la transferencia cercana y lejana, entre otras.

Complejizando aún más la situación, una creciente cantidad de investigaciones comenzó moviendo el foco de los detalles de los protocolos para ubicarlo en las diferencias individuales entre humanos ${ }^{29,53}$. Además de factores como el nivel de vulnerabilidad social, la edad o la presencia de algún trastorno cognitivo (como TDAH, TGD o demencia), se está analizando la efectividad de los protocolos de entrenamiento a la luz de aspectos como el estado previo de las FE de cada individuo o su motivación ${ }^{39}$. Al día de la fecha hay evidencias bastante sólidas que apuntan a que los individuos que más logran aprovechar la estimulación son aquellos que, previo al entrenamiento, tenían un peor estado de desarrollo de las $\mathrm{FE}^{8,26,54-57}$.

\section{Conclusiones}

La gran controversia sobre la eficacia del entrenamiento cognitivo sigue vigente dentro del área. En este trabajo intentamos mostrar distintas aristas que permiten comprender el porqué de las contradicciones existentes en la literatura. Aún queda mucho trabajo por realizar. Como en otros temas de la neurociencia educacional, ya es hora de que neurocientíficos y neurocientíficas comprendamos que el mundo real es mucho más complejo que el laboratorio; que es necesario tener en cuenta las diferencias individuales, pero sin por eso perder la rigurosidad que caracteriza a las ciencias exactas, y que debemos interactuar más, mucho más y como pares, con la comunidad educativa en su conjunto, incluyendo a niños, niñas, adolescentes, docentes y familias, para que entre todos podamos encontrar las herramientas que nos permitan educar igualando oportunidades y maximizando el potencial de cada individuo. 


\section{Referencias}

1. Friedman NP, Miyake A. Unity and diversity of executive functions: Individual differences as a window on cognitive structure. Cortex [Internet]. 2017;86:186-204. Available from: http://dx.doi.org/10.1016/j.cortex.2016.04.023

2. Blair C, Razza RP. Relating Effortful Control, Executive Function, and False Belief Understanding to Emerging Math and Literacy Ability in Kindergarten. Child Dev. 2007;78(2):647-63.

3. Brocka LL, Rimm-Kaufmana SE, Nathansona L, Grimmb KJ. The contributions of 'hot' and 'cool' executive function to children's academic achievement, learning-related behaviors, and engagement in kindergarten. Early Child Res Q. 2009;32(2 II):337-49.

4. Brown TE, Ma JML. Improvements in Executive Function Correlate with Enhanced Performance and Functioning and Health-Related Quality of Life: Evidence from Controlled Trials in ADHD Improvements in Executive Function Correlate with Enhanced Performance and Functioning and. Postgrad Med. 2010;122(5).

5. Burrage MS, Ponitz CC, McCready EA, Shah P, Sims BC, Jewkes $A M$, et al. Age- and schooling-related effects on executive functions in young children: A natural experiment. Child Neuropsychol. 2008;14(6):510-24.

6. Colé $P$, Duncan LG, Blaye A. Cognitive flexibility predicts early reading skills. Front Psychol. 2014;40(2):56.

7. Morrison FJ, Ponitz CC, McClelland MM. Self-regulation and academic achievement in the transition to school. Child Dev Intersect Emot Cogn [Internet]. 2010;(May 2014):203-24. Available from: http://content.apa.org/books/12059-011

8. Titz C, Karbach J. Working memory and executive functions: effects of training on academic achievement. Psychol Res. 2014;78(6):852-68.

9. Viterbori P, Usai MC, Traverso L, De Franchis V. How preschool executive functioning predicts several aspects of math achievement in Grades 1 and 3: A longitudinal study. J Exp Child Psychol [Internet]. 2015;140:38-55. Available from: http://dx.doi.org/10.1016/j.jecp.2015.06.014

10. Miyake A, Friedman NP. The Nature and Organization of Individual Differences in Executive Functions: Four General Conclusions. 2013;21(1):1-5.

11. Rothbart MK, Sheese BE, Posner MI. Executive Attention and Effortful Control: Linking Temperament, Brain Networks, and Genes. Child Dev Perspect. 2007;1(1):2-7.

12. Lipina S. Pobre cerebro: Los efectos de la pobreza sobre el desarrollo cognitivo y emocional, y lo que la neurociencia puede hacer para prevenirlo. 2019 [cited $2020 \mathrm{Jul}$ 7]; Available from: https://books.google.com/books?hl=es\&l$r=\& i d=-m 3 A D w A A Q B A J \& o i=f n d \& p g=P T 6 \& d q=$ sebas tian+lipina+pobre+cerebro\&ots=EIDqxIm1f7\&sig=xoQ1iyqOSEfVAdbSz21MAZw41zQ

13. McDermott JM, Westerlund A, Zeanah $\mathrm{CH}$, Nelson CA, Fox NA. Early adversity and neural correlates of executive function: Implications for academic adjustment. Dev Cogn Neurosci [Internet]. 2012;2(SUPPL. 1):S59-66. Available from: http://dx.doi.org/10.1016/j.den.2011.09.008

14. Blair C. Stress and the Development of Self-Regulation in Context. Child Dev Perspect [Internet]. 2010;23(1):1-7. Available from: https://www.ncbi.nlm.nih.gov/pmc/articles/ PMC3624763/pdf/nihms412728.pdf

15. Shields GS, Sazma MA, Yonelinas AP. The Effects of Acute Stress on Core Executive Functions: A Meta-Analysis and Comparison with Cortisol. Neurosci Biobehav Rev [Internet]. 2017;68(530):651-68. Available from: http://www.ncbi.nlm. nih.gov/pubmed/27371161\%0A http://www.pubmedcentral. nih.gov/articlerender.fcgi?artid=PMC5003767

16. Burger K. How does early childhood care and education affect cognitive development? An international review of the effects of early interventions for children from different social backgrounds. Early Child Res Q [Internet]. 2010;25(2):140-65. Available from: http://dx.doi.org/10.1016/j.ecresq.2009.11.001

17. Irwin L, Siddiqi A, Hertzman C. Early Child Development: A Powerful Equalizer. 2009;

18. Zhang Q, Wang C, Zhao Q, Yang L, Buschkuehl M, Jaeggi SM. The malleability of executive function in early childhood: Effects of schooling and targeted training. Dev Sci. 2018;(August):1-15.

19. Bryck RL, Fisher PA. Training the Brain: Practical Applications of Neural Plasticity From the Intersection of Cognitive Neuroscience, Developmental Psychology, and Prevention Science. Am Psychol. 2012;67(2):87-100.

20. Hsu NS, Novick JM, Jaeggi SM. The development and malleability of executive control abilities. Front Behav Neurosci [Internet]. 2014;8(June):1-15. Available from: http://journal. frontiersin.org/article/10.3389/fnbeh.2014.00221/abstract

21. Anguera JA, Boccanfuso J, Rintoul JL, Faraji F, Janowich J, Kong $E$, et al. Video game training enhances cognitive control in older adults J.A. Nature. 2014;501(7465):97-101.

22. Karbach J, Könen T, Spengler M. Who Benefits the Most? Individual Differences in the Transfer of Executive Control Training Across the Lifespan. J Cogn Enhanc [Internet]. 2017;1(4):394-405. Available from: http://link.springer. com/10.1007/s41465-017-0054-z

23. Klingberg $\mathrm{T}$, Forssberg $\mathrm{H}$, Westerberg $\mathrm{H}$. Training of Working Memory in Children With ADHD. J Clin Exp Neuropsychol. 2003;24(6):781-91.

24. Merzenich MM, Gabrieli JDE, Temple E, Miller SL, Tallal $P$, Deutsch GK, et al. Neural deficits in children with dyslexia ameliorated by behavioral remediation: Evidence from functional MRI. Proc Natl Acad Sci. 2003;100(5):2860-5.

25. Sitzer DI, Twamley EW, Jeste D V. Cognitive training in Alzheimer's disease: A meta-analysis of the literature. Acta Psychiatr Scand. 2006;114(2):75-90.

26. Karbach J, Verhaeghen P. Making Working Memory Work: A Meta-Analysis of Executive-Control and Working Memory Training in Older Adults. Psychol Sci. 2014;25(11):2027-37.

27. A. D. Baddeley. Working Memory. Science (80- ). 1992;302 (1110):239-435.

28. Au J, Sheehan E, Tsai N, Duncan GJ. Improving fluid intelligence with training on working memory: a meta-analysis. Psychon Bull Rev. 2014;22(2).

29. Jaeggi SM, Buschkuehl M, Jonides J, Shah P. Short- and 
long-term benefits of cognitive training. Proc Natl Acad Sci. 2011;108(25):10081-6.

30. Wang C, Jaeggi SM, Yang L, Zhang T, He X. Narrowing the achievement gap in low-achieving children by targeted executive function training. J Appl Dev Psychol [Internet]. 2019;63(July 2018):87-95. Available from: https://doi. org/10.1016/j.appdev.2019.06.002

31. Blakey E, Carroll DJ. A short executive function training program improves preschoolers' working memory. Front Psychol. 2015;6(NOV):1-8.

32. Spencer-Smith M, Klingberg T. Working Memory Training. In: The Wiley handbook of cognitive control [Internet]. 2017. p. 1199-201. Available from: http://insights.ovid.com/crossref?an=00003246-201807000-00029

33. Blair C, Raver CC. Closing the achievement gap through modification of neurocognitive and neuroendocrine function: Results from a cluster randomized controlled trial of an innovative approach to the education of children in kindergarten. PLoS One. 2014;9(11).

34. Goldin AP, Segretin MS, Hermida MJ, Paz L, Lipina SJ, Sigman $M$. Training planning and working memory in third graders. Mind, Brain, Educ. 2013;7(2):136-46

35. Nin V, Goldin AP, Carboni A. Mate Marote: Videojuegos para Estimular el Desarrollo de Procesos Cognitivos. IEEE-RITA. 2019;14:1-10

36. Goldin AP, Hermida MJ, Shalom DE, Costa ME, Lopez-Rosenfeld $M$, Segretin MS, et al. Far transfer to language and math of a short software-based gaming intervention. Proc Natl Acad Sci. 2014;54(4):311-3.

37. Diamond A, Ling DS. Conclusions about interventions, programs, and approaches for improving executive functions that appear justified and those that, despite much hype, do not. Dev Cogn Neurosci [Internet]. 2016;18:34-48. Available from: http://dx.doi.org/10.1016/j.dcn.2015.11.005

38. Owen AM, Hampshire A, Grahn J a, Stenton R, Dajani S, Burns AS, et al. Putting brain training to the test. Nature [Internet]. 2010;465(7299):775-8. Available from: http://www.nature.com/ nature/journal/v465/n7299/full/nature09042.html?lang=en

39. Jaeggi SM, Buschkuehl M, Shah P, Jonides J. The role of individual differences in cognitive training and transfer. Mem Cogn. 2014;42(3):464-80.

40. Klingberg T. Training and plasticity of working memory. Trends Cogn Sci [Internet]. 2010;14(7):317-24. Available from: http://dx.doi.org/10.1016/j.tics.2010.05.002

41. Melby-lervåg M, Redick TS, Hulme C. Working Memory Training Does Not Improve Performance on Measures of Intelligence or Other Measures of "Far Transfer ": Evidence From a Meta-Analytic Review. Perspect Psychol Sci. 2016;11(4):51234.

42. Melby-lervåg $M$, Hulme $C$. There is no convincing evidence that working memory training is effective: A reply to Au et al. (2014) and Karbach and Verhaeghen (2014). Psychon Bull Rev. 2015.
43. Schwaighofer M, Fischer F, Bühner M. Does Working Memory Training Transfer? A Meta-Analysis Including Training Conditions as Moderators. Educ Psychol. 2015;50(2).

44. Long Q, Hu N, Li H, Zhang Y, Yuan J, Chen A. Suggestion of Cognitive Enhancement Improves Emotion Regulation. Emotion. 2019;1(999):1-8.

45. Sala G, Gobet F. Working memory training in typically developing children: A meta-analysis of the available evidence. Dev Psychol. 2017;53:671-85.

46. Sala G, Aksayli ND, Tatlidil KS, Tatsumi T, Gondo Y. Near and Far Transfer in Cognitive Training: a Second-Order Meta-Analysis. Collabra Psychol. 2019;5(1):1-22.

47. Simons DJ, Boot WR, Charness N, Gathercole SE, Chabris CF, Hambrick DZ, et al. Do "Brain-Training" Programs Work? Psychol Sci Public Interes Suppl. 2016;17(3):103-86.

48. Aksayli ND, Sala G, Gobet F. The cognitive and academic benefits of Cogmed: A meta-analysis. Educ Res Rev [Internet]. 2019;27(April):229-43. Available from: https://doi. org/10.1016/j.edurev.2019.04.003

49. Stanford Center on Longevity Letter - Cognitive Training Data [Internet]. [cited 2020 May 31]. Available from: https://www. cognitivetrainingdata.org/the-controversy-does-brain-training-work/stanford-letter/

50. Cognitive Training Data Response Letter - Cognitive Training Data [Internet]. [cited 2020 May 31]. Available from: https:// www.cognitivetrainingdata.org/the-controversy-does-braintraining-work/response-letter/

51. Published Papers - Cognitive Training Data [Internet]. [cited 2020 May 31]. Available from: https://www.cognitivetrainingdata.org/studies-cognitive-training-benefits/

52. Green CS, Bavelier D, Kramer AF, Vinogradov S, Ansorge U, Ball KK, et al. Improving Methodological Standards in Behavioral Interventions for Cognitive Enhancement. J Cogn Enhanc. 2019;3(2019):2-29.

53. Albert DW, Hanson JL, Skinner AT, Dodge KA, Steinberg L, Deater-Deckard K, et al. Individual differences in executive function partially explain the socioeconomic gradient in middle- school academic achievement. Dev Sci. 2020;

54. Dorbath L, Hasselhorn M, Titz C. Aging and executive functioning: A training study on focus-switching. Front Psychol. 2011;2(OCT):1-12.

55. Best JR, Miller PH. A Developmental Perspective on Executive Function. Child Dev. 2010;81(6):1641.60.

56. Whitlock LA, Mclaughlin AC, Allaire JC. Computers in Human Behavior Individual differences in response to cognitive training: Using a multi-modal, attentionally demanding gamebased intervention for older adults. Comput Human Behav [Internet]. 2012;28(4):1091-6. Available from: http://dx.doi. org/10.1016/j.chb.2012.01.012

57. Segretin MS, Lipina SJ, Hermida MJ, Sheffield TD, Nelson JM, Espy KA, et al. Predictors of cognitive enhancement after training in preschoolers from diverse socioeconomic backgrounds. Front Psychol. 2014;5(MAR):1-21. 